\title{
Assessing $G W$ approaches for predicting core level binding energies
}

Michiel J. van Setten, ${ }^{1, *}$ Ramon Costa, ${ }^{2}$ Francesc Viñes, ${ }^{3}$ Francesc Illas ${ }^{3, \dagger}$

1) Nanoscopic Physics, Institute of Condensed Matter and Nanosciences, Université catholique de Louvain, 1348 Louvain-la-Neuve, Belgium.

2) Departament de Química Inorgànica i Orgànica \& Institut de Química Teòrica $i$ Computacional (IQTCUB), Universitat de Barcelona, Martí i Franqués 1, 08028 Barcelona, Spain.

3) Departament de Ciència de Materials i Química Fisica \& Institut de Química Teòrica i Computacional (IQTCUB), Universitat de Barcelona, Martí i Franqués 1, 08028 Barcelona, Spain.

\section{Abstract}

Here we present a systematic study on the performance of different $G W$ approaches: $G_{0} W_{0}, G_{0} W_{0}$ with linearized quasi particle equation (lin- $G_{0} W_{0}$ ), and quasiparticle selfconsistent $G W$ (qs $G W$ ), in predicting core level binding energies (CLBEs) on a series of representative molecules comparing to Kohn-Sham (KS) orbital energy based results. KS orbital energies obtained using the PBE functional are 20-30 eV lower in energy than experimental values obtained from X-ray photoemission spectroscopy (XPS), showing that any Koopmans-like interpretation of KS core level orbitals fails dramatically. Results from qs $G W$ lead to CLBEs that are closer to experimental values from XPS, yet too large. For the qs $G W$ method, the mean absolute error is about $2 \mathrm{eV}$, an order of magnitude better than plain KS PBE orbital energies and quite close to predictions from $\triangle \mathrm{SCF}$ calculations with the same functional, which are accurate within $\sim 1 \mathrm{eV}$. Smaller errors of $\sim 0.6 \mathrm{eV}$ are found for qs $G W$ CLBE shifts, again similar to those obtained using $\triangle \mathrm{SCF}$ PBE. The computationally more affordable $G_{0} W_{0}$ approximation leads to results less accurate than qs $G W$, with an error of $\sim 9 \mathrm{eV}$ for CLBEs, and $\sim 0.9 \mathrm{eV}$ for their shifts. Interestingly, starting $G_{0} W_{0}$ from PBE0 reduces this error to $\sim 4 \mathrm{eV}$ with a slight improvement on the shifts as well $(\sim 0.4 \mathrm{eV})$. The validity of the $G_{0} W_{0}$ results is however questionable since only linearized quasi particle equation results can be obtained. The present results pave the way to estimate CLBEs in periodic systems where $\triangle \mathrm{SCF}$ calculations are not straightforward although further improvement is clearly needed.

*mjvansetten@gmail.com, orcid.org/0000-0003-0557-5260

† Corresponding author: francesc.illas@ub.edu, orcid.org/0000-0003-2104-6123 
Graphic for TOC

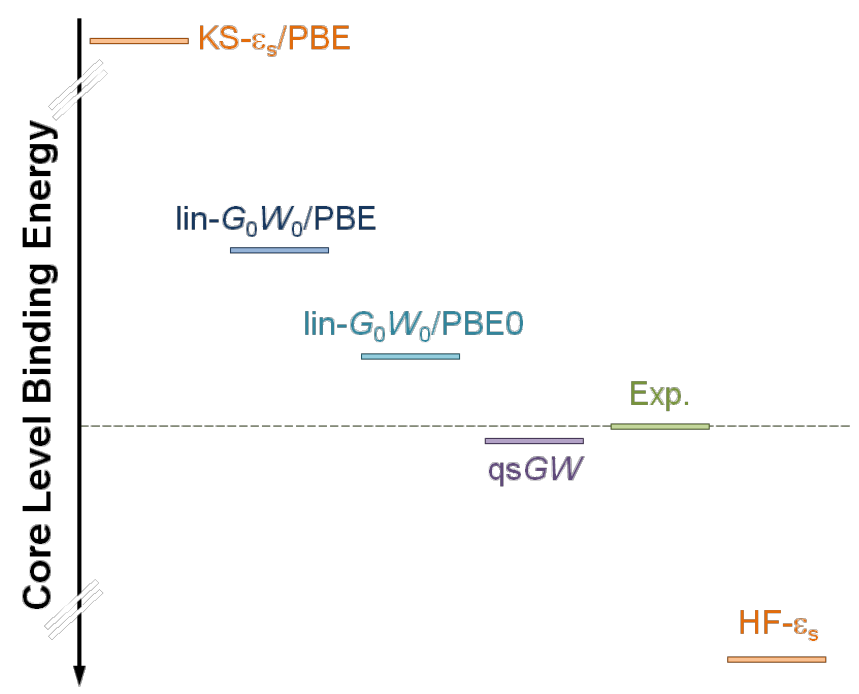




\section{Introduction}

Core level binding energies (CLBEs), experimentally accessible through X-Ray photoelectron spectroscopy (XPS), ${ }^{1}$ provide direct insight into the elemental chemistry of molecules, nanostructures, and solid materials. They contain information about the chemical environment, oxidation state, hybridization, and coordination of a given type of atom in a given sample. ${ }^{2,3}$ XPS applied to solid samples is, in addition, surface sensitive and, hence, XPS is broadly used in surface science and catalysis. Here, the assignment of XPS features is not always straightforward and assistance from theoretical calculations becomes necessary as recognized in several reviews. ${ }^{2,4}$ For instance, XPS of atomic $\mathrm{O}$ on $\mathrm{Al}(111)$ exhibit two well defined peaks that one would naively assign to $\mathrm{O}$ above and below the surface. However, theoretical analysis reveals that this hypothesis is not consistent with results from ab initio cluster model calculations, and these offer indeed an alternative explanation. ${ }^{5,6}$

The landmark paper of Bagus ${ }^{7}$ on the application of the Hartree-Fock method defines the variational determination of CLBE as a difference of the total energy between the molecule's ground state and the cation with a core hole. The resulting procedure is generally known as $\triangle \mathrm{SCF}$ since it implies the difference of two selfconsistently determined energies. Because the involved energies are obtained from a variational calculation, it turns out that, for a given method used to obtain the total energy of atoms, molecules or solids, $\triangle \mathrm{SCF}$ constitutes the best possible estimate of CLBEs. The use of Hartree-Fock (HF) to predict CLBEs has an additional advantage since the orbital energies (HF orbital energies or HF- $\varepsilon_{\mathrm{s}}$ ) fulfill the Koopman's theorem ${ }^{8}$ and, hence, the core orbital energies provide an estimate of the so-called initial state effects, those already present in the neutral molecule. The difference between the CLBEs estimated from the HF orbital energy and the $\triangle \mathrm{SCF}$ calculation is usually referred to as orbital relaxation energy, and essentially contains the response of the electron density to the presence of the core hole, usually denoted as a final state effect. ${ }^{2,3}$ Note, however, that the distinction between initial and final state is applicable to XPS main peaks only, i.e., excluding satellites and multiplets. Nevertheless, one has to point out that Hartree-Fock lacks electron correlation effects, which, although modestly contributing to the CLBEs, would need to be considered. Electron correlation effects can be explicitly included through post-Hartree-Fock methods such as configuration interaction or coupled cluster, or implicitly through the exchange- 
correlation potential in density functional theory (DFT) based methods. The latter has the additional advantage of being easily implemented for periodic systems, which is very appealing when dealing with solid materials.

In practice, DFT based methods rely on the Kohn-Sham implementation, a formalism closely resembling Hartree-Fock to the point that upon solving the so-called Kohn-Sham equations one obtains a set of single particle orbitals (KS orbitals) and energies (KS orbital energies or $\mathrm{KS}-\varepsilon_{\mathrm{s}}$ ). However, there are important differences between $\mathrm{HF}-\varepsilon_{\mathrm{S}}$ and $\mathrm{KS}-\varepsilon_{\mathrm{S}}$ since the later do not fulfill the Koopmans' theorem and, in the case of core states, the numerical values are smaller than the experimental CLBEs. Although not rigorously supported from theory, it has been claimed that conceptually $\mathrm{HF}$ and KS orbitals are similar. ${ }^{9,10}$ From this perspective, taking the $\mathrm{KS}-\varepsilon_{\mathrm{s}}$ as a measure of initial state CLBEs will lead to an unphysical positive orbital relaxation energy. ${ }^{11,12}$ The alternative view, to interpret KS orbitals as an approximation to Dyson orbitals, ${ }^{13,14}$ which, as such, would contain orbital relaxation, hence seems more physical. This point of view is developed in detail in the review papers by Ortiz ${ }^{15}$ and by Ortiz and Öhrn. ${ }^{16}$

Yet, $\mathrm{KS}-\varepsilon_{\mathrm{s}}$ become very useful when aiming at analyzing CLBE shifts ( $\left.\Delta \mathrm{CLBE}\right)$, that is, the difference between CLBEs for a given element in the system of interest and a reference value. ${ }^{9}$ For the $1 s$ core level of carbon atoms for instance, the energy ranges form 290 to about $300 \mathrm{eV}$. To distinguish between carbon atoms in similar surroundings however, an accuracy of the order of $0.1 \mathrm{eV}$ is often needed. Accurate predictions of CLBEs by means of DFT methods are well possible within a $\triangle \mathrm{SCF}$ formalism, and recent work shows that, depending on the functional used, the calculated values are of a quality similar or superior to those predicted by Hartree-Fock. ${ }^{17,18}$ However, apart from the inconvenient dependence on the choice of the functional, it is necessary to point out that $\triangle \mathrm{SCF}$ in periodic systems is problematic since it involves the use of charged unit cells and different, more or less accurate, ways to counteract this have been proposed. These range from the use of a uniform negative background that may distort the electron density, to various pseudopotential based approaches ${ }^{19}$ among which a common choice is to make use of a pseudopotential extracted from a core ionized atom ${ }^{20}$ with concomitant limitations due to constraints in the extent of core electron density relaxation. Recent work shows that these procedures are useful on determining $\triangle$ CLBEs but, unfortunately, fail to predict accurate absolute values of CLBEs. ${ }^{21}$ 
A possible way to overcome the difficulties arising from the use of DFT methods to approach CLBEs in periodic systems is to make use of the $G W$ formulation, earlier introduced by Hedin. ${ }^{22}$ The $G W$ method includes many body effects beyond the mean-field description of the electron-electron interaction in DFT via the so-called selfenergy, which is non-local and energy dependent and, in a sense, replaces the exchange correlation potential in DFT. ${ }^{23,24}$ Reviewing the $G W$ formalism here is beyond the scope of this letter and the interested reader is addressed to the excellent review of Aryasetiawan and Gunnarsson for detailed information ${ }^{25}$ All available practical implementations of $G W$ have a common feature, the need for large computational resources. This has led to different levels of approximations resulting in various flavors denoted as $G_{0} W_{0}, G W_{0}$, eigenvalue self-consistent $G W$, or quasi-particle self-consistent $G W .^{26}$ All these approximations convert the KS particles into quasiparticles (qp) with a well-defined physical meaning. In the case of occupied states, the qp energies effectively represent ionization potentials whereas in the case of unoccupied states, they represent electron affinities. In the case of periodic insulators and semiconductors $q p$ from $G W$ calculations provide an accurate estimate of the fundamental band gap of these materials ${ }^{27,28}$ and also of states associated to point defects such as F-centers in simple oxides. ${ }^{29,30}$ Apart from the high computational cost of the $G W$ calculations, one must also point out the dependence of the results with respect to the starting density, ${ }^{31}$ when the results are not obtained from a fully self-consistent approach as is the case in $G_{0} W_{0}$, lowest level of this theoretical framework. In particular, it has been suggested that hybrid functionals should provide a better starting point. ${ }^{32}$

The $G W$ method has also been applied to molecular systems and small clusters. ${ }^{33-42}$ In particular, the $G_{0} W_{0}$ level has been applied to 100 molecules (the $G W 100$ database) ${ }^{43}$ with excellent performance for the vertical ionization potential; the same database has more recently been used to benchmark different implementations and levels of self-consistency of the theory. ${ }^{44,45}$ The rather good success of $G W$ in predicting the ionization potential of molecular systems strongly suggest that it may as well provide an estimate of the CLBEs in molecules and solids where, as above commented, $\triangle \mathrm{SCF}$ calculations are cumbersome. The calculation of CLBEs obviously requires the presence of actual core levels in the calculation. Note, however, that many solid-state codes use pseudopotentials with a frozen core approximation. These make the direct calculation of CLBEs impossible although different approximations have been proposed 
to predict $\triangle$ CLBEs. ${ }^{14}$ Moreover, one must point out that $G W$ calculations for core levels may be very tricky since describing the self-energy at deep energies requires a full frequency method and solving the quasiparticle equation can be very complicated as discussed later on. The goal of the present work is precisely to investigate the performance of $G W$ on predicting CLBEs using a series of simple molecules, where experimental data and $\triangle \mathrm{SCF}$ results for several exchange-correlation functionals are available, as a convenient benchmark.

\section{Computational details.}

The ground-state calculations are based on density functional theory within the Perdew-Burke-Ernzerhof (PBE) ${ }^{46}$ generalized-gradient approximation (GGA) for the exchange-correlation functional. The molecular data set has been taken from previous work. ${ }^{17}$ The molecular structures have been fully relaxed using the PBE functional and a tight Tier 2 numerical atom-centered orbitals (NAO) basis set ${ }^{47-49}$ using the FHI-aims code. ${ }^{47}$ The quasi-particle calculations are performed with the Turbomole package using the def2-TZVP and def2-QZVP Gaussian basis sets ${ }^{50}$ and extrapolated to the complete basis-set limit. ${ }^{51}$ In addition to electron correlation, relativistic effects also play a role in determining the final value of calculated CLBEs. $2^{12,17}$ Within the $G W$ method, these can be introduced through various formalisms. ${ }^{52,53}$ Nevertheless, for sake of comparison, relativistic effects have been omitted. We note, however, that the relativistic contribution to the CLBEs is essentially atomic in nature and it increases with the atomic number. For the core levels studied in the present work contribution of relativistic effects to the $\mathrm{CLBE}$ vary from $0.13 \mathrm{eV}$ for $\mathrm{C}$ to $0.75 \mathrm{eV}$ for $\mathrm{F},{ }^{17}$ increasing along the $\mathrm{C}-\mathrm{F}$ series.

Different levels of $G W$ calculations have been carried out including the so-called one shot $G_{0} W_{0}$ approach, where both the Green's function $G$ and the screened Coulomb potential $W$ are obtained from an initial electron density arising from a given density functional approach, and the quasi particle self-consistent $G W$ (qs $G W$ ), where both $G$ and $W$ are iterated until self consistency is achieved. Clearly, qsGW results do not depend on the starting exchange-correlation functional and this has been numerically verified here. Consequently, $q_{S} G W$ results are taken here as benchmark. ${ }^{54,55}$ Note also that two different implementations of the simpler $G_{0} W_{0}$ level are used. Nevertheless, both implementations of $G_{0} W_{0}$ and $q s G W$ approaches use the full analytic expression 
from the reducible response function for the self-energy and hence the results are obtained from a full frequency method. The difference in the two $G_{0} W_{0}$ approaches used lies only in how the quasi particle equation is solved. In one case, the quasi particle equation is iteratively solved, in the other, denoted as lin- $G_{0} W_{0}$, the quasi particle equation is linearized. For the HOMO level the linearized and solved version often give very similar results. For core levels this is not the case. Due to the much larger corrections for core levels, in all molecules studied here the $G_{0} W_{0} @ \mathrm{PBE}$ self-energy has poles in the region where the quasi particle equation (QPE) needs to be solved to resolve the core-levels. A unique solution hence becomes impossible. The final $G_{0} W_{0}$ results reported in this work are all obtained from the linearized version, which circumvents this problem. Besides the PBE functional we also report lin- $G_{0} W_{0}$ starting from PBE0. For the PBE0 starting point the solving the QPE is also not uniquely possible. Only for a HF starting point this becomes feasible, see supplementary information. For a complete description of the $G W$ implementations including the two $G_{0} W_{0}$ approaches mentioned above, the reader is referred to the original work on the implementation of $G W$ in Turbomole ${ }^{56}$ reporting also result for valence ionization potentials of a broad set of molecules for $G_{0} W_{0}$, and in Kaplan et al. for qs $G W{ }^{57}$

\section{Results and discussion}

The set of molecules used to assess the reliability of the different methods considered here consists of $\mathrm{CH}_{4}, \mathrm{CF}_{4}, \mathrm{CO}_{2}, \mathrm{HCN}, \mathrm{H}_{3} \mathrm{COCH}_{3}, \mathrm{H}_{3} \mathrm{COH}, \mathrm{H}_{2} \mathrm{O}, \mathrm{NH}_{3}$, pyridine, and pyrrole. The calculations are carried out for the $\mathrm{C}, \mathrm{N}, \mathrm{O}$, and $\mathrm{F} 1 s$ core levels for which experimental data is available. For reproducibility, all molecular geometries are reported in Table S1 of the supporting information. In addition, Table S2 reports the PBE total energy at the optimized geometry corresponding to the tight Tier 2 NAO basis set. To provide insight into the quality of this basis set Table S2 also reports the PBE total energy, at the same geometry, obtained with Gaussian type orbital (GTO) basis sets of aug-cc-pCVTZ and aug-cc-pCV5Z level of quality, ${ }^{58,59}$ computed using Gaussian09. ${ }^{60}$ Inspection of Table S2 clearly shows that the tight Tier 2 NAO basis set quality is even higher than that of aug-cc-pCVTZ and only slightly below aug-ccpCV5Z.

Table 1 collects the CLBEs for the whole set of molecules as obtained from the different levels of theory together with experimental values taken from the literature. ${ }^{61,62}$ Nevertheless, one must advert that with the current implementation, fully 
analytic frequency treatment and with only a smp parallelism in TURBOMOLE, the calculation for pyridine at the def2-QZVP is not feasible and the corresponding extrapolated result could not be obtained; here the def2-TZVP value is listed instead. A quick inspection of Table 1 shows that, on average, going from KS to lin- $G_{0} W_{0}$ to qs $G W$, the calculated results are progressively approaching experiment with best results attained for the qs $G W$ method. A deeper insight into the relative errors is provided in Table 2 showing that the error for each core level is different with a mean absolute error (MAE) larger than $25 \mathrm{eV}$ for the KS- $\varepsilon_{\mathrm{s}}$ estimates, about 3-9 eV for $G_{0} W_{0}$, and slightly less than $2 \mathrm{eV}$ at the highest qs $G W$ level. These results illustrate that qs $G W$ significantly improves the agreement with experiment as compared to the KS eigenvalues, a result which is in line with previous findings concerning the first ionization potential (HOMO) in molecules. ${ }^{43,63}$ Nevertheless, the accuracy reached by qs $G W$ may not be sufficient to properly interpret XPS experiments. The present results illustrate the difficulties of the $G W$ methods on describing CLBEs. We stress that describing the self-energy at deep energies requires a full frequency method, which makes the calculations quite costly. Also, for CLBEs, $G_{0} W_{0}$ starting from the PBE functional faces fundamental problems since the self-energy has poles in the region where the quasiparticle equation is solved giving rise to a multitude of possible solutions. Besides the obvious numerical issues this grossly complicates the physical interpretation. Using the linearized quasiparticle equation solves at least the numerical part of the problem, solutions become numerically stable and unique but accuracy remains poor. In practice, it is more suitable to predict the quasiparticle energies of valence states. ${ }^{30}$ Using a hybrid functional as starting point may remediate this deficiency as shown in valence states comparing to experiment ${ }^{31}$ and also to $\operatorname{sc} G W$ calculations. ${ }^{64}$ This is because the core levels are already deeper causing the poles of the self-energy to be deeper in energy as well. Alternatively one can start from the HF density but here the lin- $G_{0} W_{0}$ leads to values, which, not surprisingly, are overestimating. The advantage is that in this case the QPE can be solved, the original poles are further away and come up in energy during the selfconsistency process. The average over overestimation is however roughly $6 \mathrm{eV}$ (see Table S4); a significant improvement over $H F-\varepsilon_{\mathrm{s}}$ again with the correct physical ingredients where final state effects are introduced by the many body terms of the $G W$ approach but a deterioration with respect to $\mathrm{G}_{0} \mathrm{~W}_{0} @ \mathrm{PBE} 0$. In the case of the qs $G W$, the poles move deeper as well and, in addition, the contribution of the off-diagonal terms of the self-energy matrix elements make it less sharply peaked. 
For CLBEs calculated using the PBE functional and employing a $\triangle \mathrm{SCF}$ approach the MAE is of $\sim 1 \mathrm{eV}$, this is larger than the $0.3-0.4 \mathrm{eV}$ corresponding values for calculations at the Hartree-Fock level or using the meta-GGA TPSS functional. ${ }^{17}$ In this sense, the MAE of $\sim 2 \mathrm{eV}$ for the qs $G W$ results is really remarkable and puts this method as a good choice to estimate CLBEs, especially in periodic solids. Here one must point out that the qs $G W$ result does not depend on the starting point, yet the fact that the accuracy reached is lower than $\triangle \mathrm{SCF}$ with PBE0 or TPSS functionals ${ }^{17}$ indicates that higher order terms are likely to be needed in the expansion of the screened potential $W$ and the self-energy.

Finally, it is also important to note that, in most practical cases, one is not interested in the absolute CLBEs but in their shifts with respect to a given reference ( $\Delta \mathrm{CLBE})$. Taking $\mathrm{CH}_{4}, \mathrm{H}_{2} \mathrm{O}$, and $\mathrm{NH}_{3}$ as references for the $\mathrm{C}(1 s), \mathrm{O}(1 s)$, and $\mathrm{N}(1 s)$, the values for the $\triangle \mathrm{CLBEs}$ arising from $\mathrm{KS}-\varepsilon_{\mathrm{s}}$, lin- $G_{0} W_{0} @ \mathrm{PBE}$, lin- $G_{0} W_{0} @ \mathrm{PBE} 0$, and qs $G W$ are reported in Table 3 and the statistic analysis of errors in Table 4. The later shows that MAE values of 0.7, 0.9, 0.4 and $0.6 \mathrm{eV}$ are found for KS- $\varepsilon_{\mathrm{s}}$, lin- $G_{0} W_{0} @ \mathrm{PBE}$, lin- $G_{0} W_{0} @ \mathrm{PBE} 0$, and qs $G W$ respectively. Note, however, that the experimental $\triangle \mathrm{CLBE}$ values seldom exceed $10 \mathrm{eV}$, which makes the relative errors still rather large. Another important feature emerges when comparing calculated and experimental $\triangle \mathrm{CLBE}$ values. Figure 1 shows that while the trends are well reproduced, there is a significant dispersion with the best values corresponding to lin- $G_{0} W_{0} @ \mathrm{PBE} 0$, and qs $G W$ respectively.

\section{Conclusions}

A study has been carried out to assess the performance of different $G W$ approaches in predicting core level binding energies (CLBEs). Results presented for a series of representative molecules allows us to establish some first conclusions.

First, the present calculations agree with previous studies ${ }^{9,10}$ showing that the Kohn-Sham orbital energies cannot be taken as a measure of the CLBEs and they do not represent an estimate of initial state effects. The Kohn-Sham orbital energies are always smaller than experimental CLBEs with differences with respect to experiment in the 20$30 \mathrm{eV}$ range. Interpreting them as approximate initial states would hence imply unphysical positive relaxation energies. In the framework of DFT, initial state effects can be recovered by computing the molecular system with a core hole with the fixed 
electron density of neutral molecule as discussed in previous works ${ }^{10}$ and will not be further commented here. The interpretation of the KS-orbitals as approximate Dyson orbitals hence seems more physical especially for core levels.

The most accurate results, as compared to experiment, are obtained at the quasiparticle self-consistent $G W$ level (qs $G W$ ). Nevertheless, to achieve good agreement with experiment, extrapolation to the complete basis set limit is needed, especially for the absolute CLBE although it may be less problematic for the shifts. In any case, the rather good agreement with experiment evidences that $G W$ is able to largely correct the failure of the KS and HF orbital energies covering a large part of relaxation energy, thus going in the direction of the right-answer for the right-reason.

We find that the qs $G W$ calculated CLBEs are always larger than the experimental values, as expected due to underscreening. The mean absolute error with respect to experiment is reduced to about $2 \mathrm{eV}$; one order of magnitude smaller than for $\mathrm{KS}$ values and close to predictions from variational $\triangle \mathrm{SCF}$ calculations which, for the PBE functional, are in the $1 \mathrm{eV}$ range. Here it is important to point out that an absolute error of $2 \mathrm{eV}$ on a quantity in the $300-500 \mathrm{eV}$ range implies a percent error of $0.6 \%$ only, which is quite remarkable, and, actually, very similar to the percent error of $0.5 \%$ achieved when considering the qs $G W$ prediction of the first ionization potential. ${ }^{43}$ a somehow larger error (6.5\%) is obtained in the case of lin- $\mathrm{G}_{0} \mathrm{~W}_{0} @ \mathrm{HF}$. Note also that adding contribution from relativistic effects will further decrease the $\triangle \mathrm{SCF}$ error to less than $1 \mathrm{eV}$. Yet, the error bar of XPS, especially when synchrotron radiation is used, can be as small as $0.1 \mathrm{eV}$, meaning that further developments are needed. Smaller errors of roughly $0.6 \mathrm{eV}$ are found for the $\mathrm{qs} G W$ calculated core level binding energy shifts $(\triangle \mathrm{CLBE})$ although these are surprisingly larger than those arising from Kohn-Sham orbital energies. This is an indication that $\mathrm{qs}_{\mathrm{S}} G W$ effects on same core level in different molecules are not equally taken into account.

The simpler $G_{0} W_{0}$ and computationally more affordable level of the theory also leads to a significant overall improvement with respect to KS orbital energies. However, starting from the PBE density, the calculated CLBEs are still smaller than experiment evidencing limitations inherent to this level of approximation as commented in the previous section. The absolute mean errors $(8.98$ and $3.76 \mathrm{eV}$ for the PBE and PBE0 starting points respectively) are significantly larger than the corresponding value for 
qs $G W$ but smaller than the one arising from the direct use of KS orbital energies. A paired T-test indeed proves that the differences are significant.

To summarize, CLBEs derived from $G W$ approaches represent a considerable improvement with respect to KS energies although the accuracy reached with the present implementations, even for the qs $G W$, is still lower than the one obtained from $\Delta$ SCF calculations. The simpler $G_{0} W_{0}$ method applied on top of the PBE density also lead to results improved with respect to KS predictions but with too large errors. These errors are considerably reduced when starting the $G_{0} W_{0}$ calculations from a density obtained from a hybrid functional. Similar considerations apply to the core level binding energy shifts with rather satisfactory results for the lin- $G_{0} W_{0} @ \mathrm{PBE} 0$ and qs $G W$ methods. For practical applications in computational materials science, $G_{0} W_{0}$ on top of single point $\mathrm{PBE} 0$ density at the $\mathrm{PBE}$ optimized structure may provide a practical approach since this will be computationally less demanding than going to the qs $G W$ level and lead to similar accuracy.

\section{Acknowledgements}

This research was supported by the Spanish MINECO/FEDER CTQ2015-64618-R grant and, in part, by Generalitat de Catalunya (grants 2014SGR97 and XRQTC) and by the NOMAD Center of Excellence project, which received funding from the European Union's Horizon 2020 research and innovation programme under grant agreement No 676580. F.V. thanks MINECO for a postdoctoral Ramón y Cajal (RyC) research contract (RYC-2012-10129) and F.I. acknowledges additional support from the 2015 ICREA Academia Award for Excellence in University Research. We also thank the Céci facilities funded by F.R.S-FNRS (Grant No. 2.5020.1) and Tier-1 supercomputer of the Fédération Wallonie-Bruxelles funded by the Walloon Region (Grant No. 1117545).

The authors are very grateful to Dr. Fabio Caruso of Humboldt-Universität zu Berlin (Germany) and Prof. Patrick Rinke of Aalto University in Helsinki (Finland) for their many helpful discussions regarding the $G_{0} W_{0}$ methods and their implementations in FHI-AIMS computer program package. Mrs. Helena Muñoz Galan is also acknowledged for helping to carry out some of the preliminary, not reported here, calculations. 


\section{Supporting information}

The Supporting Information is available free of charge on the ACS Publications website at DOI: $\operatorname{xxxxxxxxxxxxx}$

Table S1. Cartesian coordinates of the molecules studied in the present work as optimized FHI-AIMS tight Tier 2 PBE.

Table S2. Total PBE energy $(\mathrm{eV})$ of the molecules studied in the present work with geometries as in Table S1 and corresponding to numerical atomic orbital (NAO) tight Tier 2 basis set using the aims code and using the FHI-AIMS code and to the aug-ccpCVTZ and aug-cc-pCV5Z gaussian type orbitals (GTO) basis sets as obtained with the Gaussian09 code.

Table S3. Total PBE energy (eV) of the molecules studied in the present work with geometries as in Table S1 as obtained with the def2-VPTZ GTO basis and different codes.

Table S4. CLBEs as obtained from lin- $G_{0} W_{0} @ H F$. 
Table 1. Core level binding energies as obtained from KS, lin- $G_{0} W_{0} @ \mathrm{PBE}$, lin$G_{0} W_{0} @$ PBE0, and quasi particle self-consistent (qs $G W$ ) methods starting from PBE. All values correspond to a linear extrapolation of the def2-TZVP and def2-QZVP results to the complete basis set limit. Experimental values (positive as they correspond to ionization processes) are provided for comparison.

\begin{tabular}{|c|c|c|c|c|c|c|}
\hline Core level & Molecule & $\mathrm{KS}$ & $\begin{array}{c}\operatorname{lin}-G_{0} W_{0} \\
\text { @ } \mathrm{PBE}\end{array}$ & $\begin{array}{c}\operatorname{lin}-G_{0} W_{0} \\
\text { @ PBE0 }\end{array}$ & $\mathrm{qs} G W$ & Experiment \\
\hline \multirow{7}{*}{$C(1 s)$} & $\mathrm{CH}_{4}$ & -268.83 & -284.10 & -287.82 & -293.54 & 290.90 \\
\hline & $\mathrm{CF}_{4}$ & -277.89 & -292.18 & -299.39 & -302.84 & 301.80 \\
\hline & $\mathrm{CO}_{2}$ & -273.69 & -287.48 & -294.40 & -298.78 & 297.91 \\
\hline & $\mathrm{HCN}$ & -270.41 & -286.13 & -290.45 & -294.67 & 293.50 \\
\hline & $\mathrm{H}_{3} \mathrm{COCH}_{3}$ & -269.96 & -284.99 & -289.14 & -294.13 & 292.30 \\
\hline & $\mathrm{H}_{3} \mathrm{COH}$ & -269.96 & -286.15 & -291.14 & -294.69 & 292.30 \\
\hline & Pyrrole & -268.87 & -282.88 & -286.78 & -293.02 & 290.80 \\
\hline \multirow{4}{*}{$\mathrm{O}(1 \mathrm{~s})$} & $\mathrm{H}_{2} \mathrm{O}$ & -510.09 & -529.45 & -534.90 & -542.13 & 539.70 \\
\hline & $\mathrm{CO}_{2}$ & -512.42 & -530.41 & -536.28 & -542.46 & 541.30 \\
\hline & $\mathrm{H}_{3} \mathrm{COH}$ & -510.13 & -528.72 & -534.28 & -541.12 & 538.90 \\
\hline & $\mathrm{H}_{3} \mathrm{COCH}_{3}$ & -510.35 & -528.15 & -533.24 & -540.77 & 539.03 \\
\hline \multirow{4}{*}{$\mathrm{N}(1 \mathrm{~s})$} & $\mathrm{NH}_{3}$ & -379.82 & -397.04 & -401.75 & -407.84 & 405.60 \\
\hline & $\mathrm{HCN}$ & -381.18 & -397.38 & -402.21 & -408.78 & 406.80 \\
\hline & Pyridine & -380.51 & -395.24 & -400.66 & $-407.4^{*}$ & 404.90 \\
\hline & Pyrrole & -381.74 & -397.10 & -402.31 & -408.11 & 406.10 \\
\hline $\mathrm{F}(1 \mathrm{~s})$ & $\mathrm{CF}_{4}$ & -661.79 & -683.19 & -689.26 & -696.15 & 692.40 \\
\hline
\end{tabular}

*) def2-TZVP value; see text 
Table 2. Mean absolute error (MAE) for the core level binding energies reported in Table 1 and obtained from KS, lin- $G_{0} W_{0} @ \mathrm{PBE}$, lin- $G_{0} W_{0} @ \mathrm{PBE} 0$, and quasi particle self-consistent (qs $G W$ ) methods

\begin{tabular}{|c|c|c|c|c|}
\hline \multicolumn{5}{|c|}{ MAE } \\
\hline Core level & KS & $\begin{array}{c}\text { lin- } G_{0} W_{0} \\
@ \text { PBE }\end{array}$ & $\begin{array}{c}\text { lin- } G_{0} W_{0} \\
@ \text { PBE0 }\end{array}$ & qs $G W$ \\
\hline $\mathrm{C}(1 \mathrm{~s})$ & 22.84 & 7.94 & 2.91 & 1.74 \\
\hline $\mathrm{O}(1 \mathrm{~s})$ & 28.99 & 10.55 & 5.06 & 1.89 \\
\hline $\mathrm{N}(1 \mathrm{~s})$ & 25.04 & 9.16 & 4.12 & 2.08 \\
\hline TOTAL & 25.41 & 8.98 & 3.76 & 1.98 \\
\hline
\end{tabular}


Table 3. $\triangle \mathrm{CLBEs}$ as obtained from KS, lin- $G_{0} W_{0} @ \mathrm{PBE}$, lin- $G_{0} W_{0} @ \mathrm{PBE} 0$, and quasi particle self-consistent (qs $G W$ ) methods starting from $\mathrm{PBE}$, using $\mathrm{CH}_{4}, \mathrm{H}_{2} \mathrm{O}$, and $\mathrm{NH}_{3}$ as reference for $\mathrm{C}(1 \mathrm{~s}), \mathrm{O}(1 \mathrm{~s})$, and $\mathrm{N}(1 \mathrm{~s})$ core levels. All values are given in $\mathrm{eV}$

\begin{tabular}{|c|c|c|c|c|c|c|}
\hline Core level & Molecule & $\mathrm{KS}$ & $\begin{array}{c}\operatorname{lin}-G_{0} W_{0} \\
@ \mathrm{PBE}\end{array}$ & $\begin{array}{l}\operatorname{lin}-G_{0} W_{0} \\
\text { @ } \mathrm{PBE} 0\end{array}$ & $\mathrm{qs} G W$ & Experiment \\
\hline \multirow{7}{*}{$C(1 s)$} & $\mathrm{CH}_{4}$ & 0.00 & 0.00 & 0.00 & 0.00 & 0.00 \\
\hline & $\mathrm{CF}_{4}$ & -9.06 & -8.08 & -11.57 & -9.30 & -10.90 \\
\hline & $\mathrm{CO}_{2}$ & -4.86 & -3.38 & -6.58 & -5.24 & -7.01 \\
\hline & $\mathrm{HCN}$ & -1.58 & -2.03 & -2.63 & -1.13 & -2.60 \\
\hline & $\mathrm{H}_{3} \mathrm{COCH}_{3}$ & -1.13 & -0.89 & -1.32 & -0.59 & -1.40 \\
\hline & $\mathrm{H}_{3} \mathrm{COH}$ & -1.13 & -2.05 & -3.32 & -1.15 & -1.40 \\
\hline & Pyrrole & -0.04 & 1.22 & 1.04 & 0.52 & 0.10 \\
\hline \multirow{4}{*}{$\mathrm{O}(1 \mathrm{~s})$} & $\mathrm{H}_{2} \mathrm{O}$ & 0.00 & 0.00 & 0.00 & 0.00 & 0.00 \\
\hline & $\mathrm{CO}_{2}$ & -2.33 & -0.96 & -1.38 & -0.33 & -1.60 \\
\hline & $\mathrm{H}_{3} \mathrm{COH}$ & -0.04 & 0.73 & 0.62 & 1.01 & 0.80 \\
\hline & $\mathrm{H}_{3} \mathrm{COCH}_{3}$ & -0.26 & 1.30 & 1.66 & 1.36 & 0.67 \\
\hline \multirow{4}{*}{$\mathrm{N}(1 \mathrm{~s})$} & $\mathrm{NH}_{3}$ & 0.00 & 0.00 & 0.00 & 0.00 & 0.00 \\
\hline & $\mathrm{HCN}$ & -1.36 & -0.34 & -0.46 & -0.94 & -1.20 \\
\hline & Pyridine & -0.69 & 1.80 & 1.09 & - & 0.70 \\
\hline & Pyrrole & -1.92 & -0.06 & -0.56 & -0.27 & -0.50 \\
\hline
\end{tabular}


Table 4. Mean absolute error (MAE) for the $\triangle$ CLBEs reported in Table 3 and obtained from KS, lin- $G_{0} W_{0} @ \mathrm{PBE}$, lin- $G_{0} W_{0} @ \mathrm{PBE} 0$, and quasi particle self-consistent (qs $G W$ ) methods.

\begin{tabular}{|c|c|c|c|c|}
\hline \multicolumn{5}{|c|}{ MAE } \\
\hline Core level & KS & $\begin{array}{c}\text { lin- } G_{0} W_{0} \\
@ \text { PBE }\end{array}$ & $\begin{array}{c}\text { lin- } G_{0} W_{0} \\
@ \text { PBE0 }\end{array}$ & qs $G W$ \\
\hline $\mathrm{C}(1 \mathrm{~s})$ & 0.81 & 1.33 & 0.58 & 0.90 \\
\hline $\mathrm{O}(1 \mathrm{~s})$ & 0.63 & 0.34 & 0.35 & 0.54 \\
\hline $\mathrm{N}(1 \mathrm{~s})$ & 0.74 & 0.60 & 0.30 & 0.16 \\
\hline TOTAL & 0.74 & 0.87 & 0.44 & 0.64 \\
\hline
\end{tabular}


Figure 1.- Calculated KS, lin- $G_{0} W_{0} @ \mathrm{PBE}$, lin- $G_{0} W_{0} @ \mathrm{PBE} 0$, and quasi particle selfconsistent (qs $G W$ ) versus experimental core level binding energy shifts ( $\triangle \mathrm{CLBE}$ ). The dashed line corresponds to a perfect agreement.

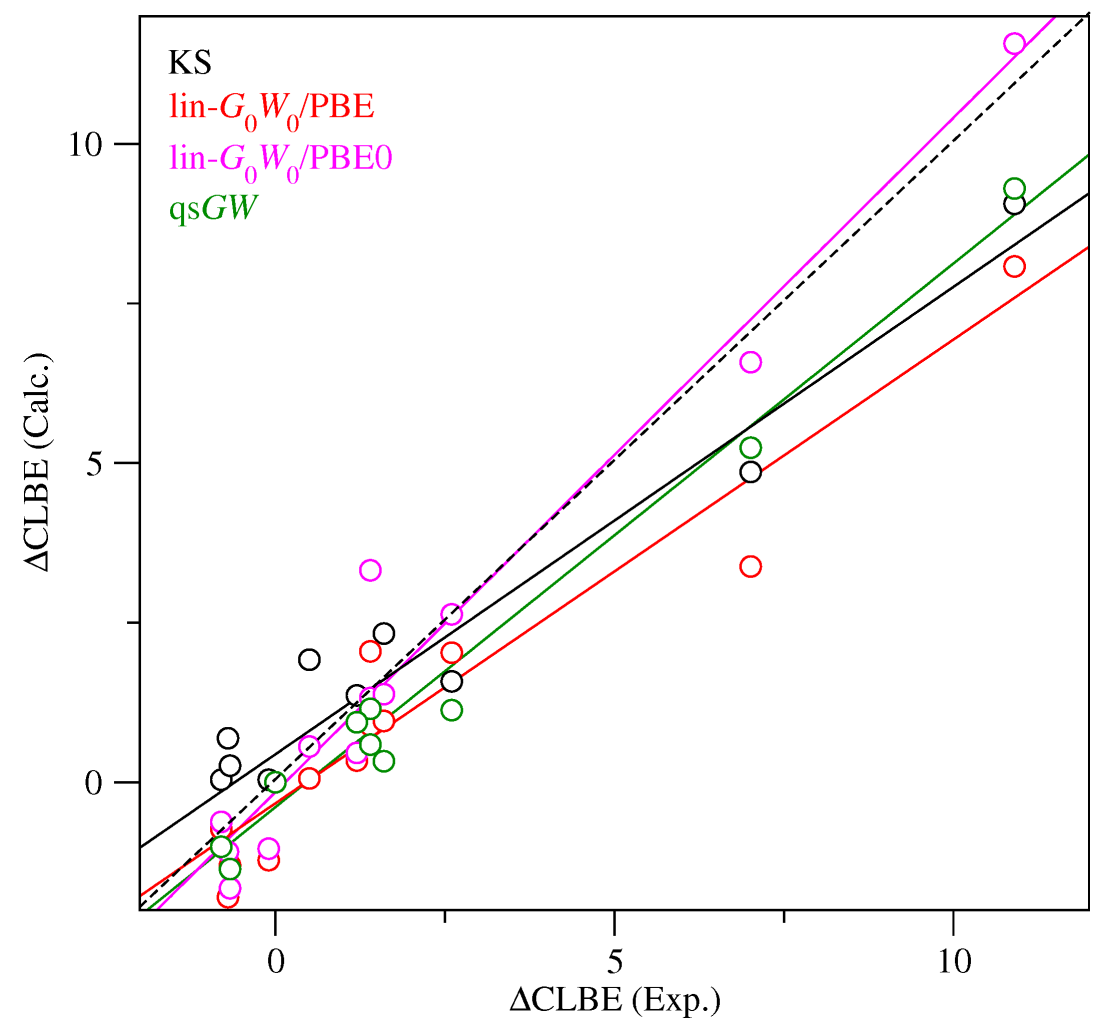




\section{References}

1 Barr, T.L. The Principles and Practice of X-Ray Photoelectron Spectroscopy. Taylor and Francis, London, 1994.

2 Bagus, P.S.; Ilton, E.S.; Nelin C.J. The interpretation of XPS spectra: Insights into materials properties. Surf. Sci. Rep. 2013, 68, 273-304.

3 Bagus, P.S.; Illas, F.; Pacchioni, G.; Parmigiani, F. Mechanisms responsible for chemical shifts of core-level binding energies and their relationship to chemical bonding. J. Electr. Spectrosc. Relat. Phenom. 1999, 100, 215-236.

4 Egelhoff, W.F. Core-level binding-energy shifts at surfaces and in solids. Surf. Sci. Rep. 1987, 6, 253-415.

5 Bagus, P.S.; Parmigiani, F.; Polzonetti, G..; Illas, F.; Brundle, C. R. X-ray photoelectron spectroscopy of oxygen adsorbates on $\mathrm{Al}(111)$ Theory and experiment. J. Vac. Sci. Technol. A 1991, 9, 1747-1748.

6 Bagus, P.S.; Brundle, C.R.; Illas, F.; Parmigiani, F.; Polzonetti, G. Evidence for oxygen island formation on $\mathrm{Al}(111)$ Cluster model theory and X-ray photoelectron spectroscopy. Phys. Rev. B 1991, 44, 9025-9034.

7 Bagus, P.S. Self-Consistent-Field Wave Functions for Hole States of Some NeLike and Ar-Like Ions. Phys. Rev. 1965, 139, 619-634.

8 Koopmans, T. Über die Zuordnung von Wellenfunktionen und Eigenwerten zu den Einzelnen Elektronen eines Atoms. Physica 1933, 1, 104-113.

9 Baerends, E.J.; Gritsenko, O.V. A Quantum Chemical View of Density Functional Theory. J. Phys. Chem. A 1997, 101, 5383-5403.

10 Stowasser, R.; Hoffmann, R. What Do the Kohn-Sham Orbitals and Eigenvalues Mean? J. Am. Chem. Soc. 1999, 121, 3414-3420.

11 Pueyo Bellafont, N.; Illas, F.; Bagus, P. S. Validation of Koopmans' theorem for density functional theory binding energies. Phys. Chem. Chem. Phys. 2015, 17, 4015-4019 
12 Pueyo Bellafont, N.; Bagus, P. S.; Illas, F. Prediction of core level binding energies in density functional theory: Rigorous definition of initial and final state contributions and implications on the physical meaning of Kohn-Sham energies. $J$. Chem. Phys. 2015, 142, 214102 (1-12).

Duffy P.; Chong D.P.; Casida, M.E.; Salahub, D.R. Assessment of Kohn-Sham density-functional orbitals as approximate Dyson orbitals for the calculation of electron-momentum-spectroscopy scattering cross sections. Phys. Rev. A 1994, 50, 4707-4728.

Gritsenko, O.V.; Baerends, E.J. The analog of Koopmans' theorem in spin-density functional theory. J. Chem. Phys. 2002, 117, 9154-9159.

Ortiz, J.V. Brueckner Orbitals, Dyson Orbitals, and Correlation Potentials. Int. J. Quantum Chem. 2004, 100, 1131-1135.

16 Öhrn, Y.; Ortiz, J. V. The electron propagator. Mol. Phys. 2010, 108, 2871-2875 and references therein

17 Pueyo Bellafont, N.; Viñes, F.; Illas, F. Performance of the TPSS Functional on Predicting Core Level Binding Energies of Main Group Elements Containing Molecules: A Good Choice for Molecules Adsorbed on Metal Surfaces. J. Chem. Theory and Comput. 2016, 12, 324-331.

18 Pueyo Bellafont, N.; Álvarez Saiz, G.; Viñes, F.; Illas, F. Performance of Minnesota functionals on predicting core-level binding energies of molecules containing main-group elements. Theor. Chem. Acc. 2016, 135, 35-43.

Pehlke, E; Scheffler, M. Evidence for Site-Sensitive Screening of Core Holes at the Si And Ge (001) Surface. Phys. Rev. Lett. 1993, 71, 2338-2341. Köhler, L.; Kresse, G. Density functional study of CO on Rh(111). Phys. Rev. B 2004, 70, 165405.

Pueyo Bellafont, N.; Viñes, F.; Hieringer, W.; Illas, F. Accurate Prediction of Core Level Binding Energies Shifts: Suitability of Projector Augmented Wave Approach as implemented in VASP. J. Comput. Chem. 2017, 38, 518-522. 
22 Hedin, L. New Method for Calculating the One-Particle Green's Function with Application to the Electron-Gas Problem. Phys. Rev. 1965, 139, A796-A822.

23 Strinati, G.; Mattausch, H. J.; Hanke, W. Dynamical Correlation Effects on the Quasiparticle Bloch States of a Covalent Crystal. Phys. Rev. Lett. 1980, 45, 290294.

24 Hybertsen, M. S.; Louie, S. G. Electron Correlation in Semiconductors and Insulators: Band Gaps and Quasiparticle Energies. Phys. Rev. B 1986, 34, 53905413.

25 Aryasetiawan, F.; Gunnarsson, O. The GW method. Rep. Prog. Phys. 1998, 61, 237-312.

26 Faleev, S.; van Schilfgaarde, M.; Kotani, T. All-electron self-consistent GW approximation: Application to $\mathrm{Si}, \mathrm{MnO}$, and $\mathrm{NiO}$. Phys. Rev. Lett. 2004, 93, $126406(1-4)$.

27 Kang, W.; Hybertsen, M. S. Quasiparticle and Optical Properties of Rutile and Anatase $\mathrm{TiO}_{2}$. Phys. Rev. B 2010, 82, 085203 (1-11).

Zhu, T.; Gao, S. P. The Stability, Electronic Structure, and Optical Property of $\mathrm{TiO}_{2}$ Polymorphs. J. Phys. Chem. C, 2014, 118, 11385-11396.

Rinke, P.; Schleife, A.; Kioupakis, E.; Janotti, A.; Rödl, C.; Bechstedt, F.; Scheffler, M.; Van de Walle, C.G. First-principles optical spectra for F centers in MgO. Phys. Rev. Lett. 2012, 108, 126404 (1-5).

Tosoni, S.; Fernandez Hevia, D.; Perez Peña, J.; Illas, F. Accurate description of optical absorption of F centers from quasiparticle band structure calculations. Phys. Rev. B 2012, 85, 115114 (1-4).

31 Liao P.; Carter, E.A. Testing variations of the GW approximation on strongly correlated transition metal oxides: hematite $\left(\alpha-\mathrm{Fe}_{2} \mathrm{O}_{3}\right)$ as a benchmark. Phys. Chem. Chem. Phys. 2011, 13, 15189-15199. 
32 Ren, X.; Rinke, P.; Scheffler, M. Exploring the Random Phase Approximation: Application to CO adsorbed on $\mathrm{Cu}(111)$. Phys. Rev. B 2009, 80, 045402 (1-8).

33 Ishii, S.; Ohno, K.; Kawazoe, Y.; Louie, S. G. Ab Initio GW Quasiparticle Energies of Small Sodium Clusters by an All-electron Mixed-basis Approach Phys. Rev. B 2001, 63, 155104 (1-6).

34 Tiago, M. L.; Chelikowsky, J. R. Optical Excitations in Organic Molecules, Clusters, and Defects Studied by First-Principles Green's Function Methods. Phys. Rev. B 2006, 73, 205334 (1-19)

35 Bruneval, F. GW Approximation of the Many-Body Problem and Changes in the Particle Number. Phys. Rev. Lett. 2009, 103, 176403 (1-4).

36 Rostgaard, C.; Jacobsen, K. W.; Thygesen, K. S. Fully Self-consistent GW Calculations for Molecules. Phys. Rev. B 2010, 81, 085103 (1-10).

37 Baumeier, B.; Andrienko, D.; Ma, Y.; Rohlfing, M. Excited States of Dicyanovinyl-Substituted Oligothiophenes from Many-Body Green's Functions Theory. J. Chem. Theory Comput. 2012, 8, 997-1002.

38 Caruso, F.; Rinke, P.; Ren, X.; Scheffler, M.; Rubio, A. Unified Description of Ground and Excited States of Finite Systems: The Self-consistent GW Approach. Phys. Rev. B 2012, 86, 081102 (1-5).

Faber, C.; Boulanger, P.; Duchemin, I.; Attaccalite, C.; Blase, X. Many-body Green's Function GW and Bethe-Salpeter Study of the Optical Excitations in a Paradigmatic Model Dipeptide. J. Chem. Phys. 2013, 139, 194308 (1-10).

Govoni, M.; Galli, G. Large Scale GW Calculations. J. Chem. Theory Comput. 2015, 11, 2680-2696.

41 Laflamme Janssen, J.; Rousseau, B.; Cote, M. Efficient Dielectric Matrix Calculations Using the Lanczos Algorithm for Fast Many-body $\mathrm{G}_{0} \mathrm{~W}_{0}$ Implementations. Phys. Rev. B 2015, 91, 125120 (1-17). 
42 Maggio, E.; Kresse, G. GW Vertex Corrected Calculations for Molecular Systems. J Chem Theory Comput. 2017, 13, 4765-4778.

43 van Setten, M. J.; Caruso, F.; Sharifzadeh, S.; Ren, X.; Scheffler, M.; Liu, F.; Lischner, J.; Lin, L.; Deslippe, J. R.; Louie, S. G.; Yang, C.; Weigend, F.; Neaton, J. B.; Evers, F.; Rinke, P. GW100: Benchmarking $\mathrm{G}_{0} \mathrm{~W}_{0}$ for Molecular Systems. $J$. Chem. Theory Comput. 2015, 11, 5665-5687.

44 Caruso, F.; Dauth, M.; van Setten, M.J.; Rinke, P. Benchmark of GW Approaches for the GW100 Test Set. J. Chem. Theory Comput. 2016, 12, 5076-5087.

45 Maggio, E.; Liu, P.; van Setten, M.J.; Kresse, G. GW100: A Plane Wave Perspective for Small Molecules. J. Chem. Theory Comput. 2017, 13, 635-648. Simple. Phys. Rev. Lett. 1996, 77, 3865-3868.

Blum, V.; Gehrke, R.; Hanke, F.; Havu, P.; Havu, V.; Ren, X.; Reuter, K.; Scheffler, M. Ab Initio Molecular Simulations with Numeric Atom-centered Orbitals. Comput. Phys. Commun. 2009, 180, 2175-2196.

Havu, V.; Blum, V.; Havu, P.; Matthias S. Efficient O(N) Integration for Allelectron Electronic Structure using Numerically Tabulated Basis Functions. $J$. Comput. Phys. 2009, 228, 8367-8379.

49 Ren, X.; Rinke, P.; Blum, V.; Wieferink, J.; Tkatchenko, A.; Sanfilippo, A.; Reuter, K.; Scheffler, M. Resolution-of-identity Approach to Hartree-Fock, Hybrid Density Functionals, RPA, MP2, and GW with Numeric Atom-centered Orbital Basis Functions. New J. Phys. 2012, 14, 053020 (1-55).

Weigend, F.; Ahlrichs, R. Balanced basis sets of split valence, triple zeta valence and quadruple zeta valence quality for $\mathrm{H}$ to $\mathrm{Rn}$ : Design and assessment of accuracy. Phys. Chem. Chem. Phys. 2005, 7, 3297-3305.

51 TURBOMOLE V7.0 2015, a development of University of Karlsruhe and Forschungszentrum Karlsruhe GmbH, 1989-2007, TURBOMOLE GmbH, since 2007. Available from http://www.turbomole.com 
52 Kühn, M.; Weigend, F. One-Electron Energies from the Two-Component $G W$ Method. J. Chem. Theory Comput. 2015, 11, 969- 979.

53 Scherpelz, P.; Govoni, M.; Hamada, I.; Galli, G. Implementation and Validation of Fully Relativistic $G W$ Calculations: Spin-Orbit Coupling in Molecules, Nanocrystals, and Solids. J. Chem. Theory Comput. 2016, 12, 3523-3544.

van Schilfgaarde, M.; Kotani, T.; Faleev, S. Quasiparticle Self-Consistent $G W$ Theory. Phys. Rev. Lett. 2006, 96, 226402 (1-4).

Kotani, T.; Van Schilfgaarde, M.; Faleev, S. V. Quasiparticle self-consistent $G W$ method: A basis for the independent-particle approximation. Phys. Rev. B 2007, 76, $165106(1-24)$.

van Setten, M. J.; Weigend, F.; Evers, F. The GW-Method for Quantum Chemistry Applications: Theory and Implementation. J. Chem. Theory Comput. 2013, 9, 232246.

Kaplan, F.; Seiler, C.; Weigend, F.; Evers, F.; van Setten, M.J. Quasiparticleselfconsistent GW for molecules. J. Chem. Theory Comput. 2016, 12, 2528-2541.

Feller, D. The Role of Databases in Support of Computational, Chemistry Calculations. J. Comp. Chem. 1996, 17, 1571-1586.

Schuchardt, K.L.; Didier, B.T.; Elsethagen, T.; Sun, L.; Gurumoorthi, V.; Chase, J.; Li, J.; Windus, T.L. Basis set exchange: a community database for computational sciences. J. Chem. Inf. Model. 2007, 47, 1045-1052.

Gaussian 09, Revision A.02. Frisch, M. J.; Trucks, G. W.; Schlegel, H. B.; Scuseria, G. E.; Robb, M. A.; Cheeseman, J. R.; Scalmani, G.; Barone, V.; Mennucci, B.; Petersson, G. A.; Nakatsuji, H.; Caricato, M.; Li, X.; Hratchian, H. P.; Izmaylov, A. F.; Bloino, J.; Zheng, G.; Sonnenberg, J. L.; Hada, M.; Ehara, M.; Toyota, K.; Fukuda, R.; Hasegawa, J.; Ishida, M.; Nakajima, T.; Honda, Y.; Kitao, O.; Nakai, H.; Vreven, T.; Montgomery, J. A., J.; Peralta, J. E.; Ogliaro, F.; Bearpark, M.; Heyd, J. J.; Brothers, E.; Kudin, K. N.; Staroverov, V. N.; Kobayashi, R.; Normand, J.; Raghavachari, K.; Rendell, A.; Burant, J. C.; Iyengar, S. S.; Tomasi, J.; Cossi, M.; Rega, N.; Millam, J. M.; Klene, M.; Knox, J. E.; Cross, J. B.; Bakken, V.; Adamo, C.; Jaramillo, J.; Gomperts, R.; Stratmann, R. E.; 
Yazyev, O.; Austin, A. J.; Cammi, R.; Pomelli, C.; Ochterski, J. W.; Martin, R. L.; Morokuma, K.; Zakrzewski, V. G.; Voth, G. A.; Salvador, P.; Dannenberg, J. J.; Dapprich, S.; Daniels, A. D.; Farkas, Ö.; Foresman, J. B.; Ortiz, J. V.; Cioslowski, J.; Fox, D. J. Wallingford CT 2009.

61 Bakke, A.A.; Chen, H.W.; Jolly, W.L. A table of absolute core-electron bindingenergies for gaseous atoms and molecules. J. Electron Spectrosc. Relat. Phenom. 1980, 20, 333-366.

62 Myrseth, V.; Saethre, L.J.; Borve, K.J.; Darrah Thomas, T. The Substituent Effect of the Methyl Group. Carbon 1s Ionization Energies, Proton Affinities, and Reactivities of the Methylbenzene. J. Org. Chem. 2007, 72, 5715-5723.

63 Rostgaard, C.; Jacobsen, K. W.; Thygesen, K. S. Fully Self-consistent GW Calculations for Molecules. Phys. Rev. B 2010, 81, 085103.

64 Gao, S. P. Cubic, Wurtzite, and 4H-BN Band Structures Calculated using GW Methods and Maximally Localized Wannier Functions Interpolation. Comput. Mater. Sci. 2012, 61, 266-269. 